\title{
CAVITATION OF THE GHON FOCUS IN AN HIV-INFECTED INFANT WHO ACQUIRED TUBERCULOSIS AFTER THE INITIATION OF HAART
}

\author{
S Innes', $M B B C h, M R C P C H$ \\ H S Schaaf ${ }^{2}, M B C h B, M M e d(P e d), D C M, M D$ (Ped) \\ M F Cotton ${ }^{1,2}, M B C h B, M M e d, P h D, F C P a e d, D T M \& H, D C H$ (SA) \\ ${ }^{1}$ KID-CRU (Children's Infectious Diseases Clinical Research Unit), Tygerberg Academic Hospital, Tygerberg, W Cape \\ ${ }^{2}$ Department of Paediatrics and Child Health, Tygerberg Academic Hospital and Stellenbosch University, Tygerberg
}

\begin{abstract}
Tuberculosis immune reconstitution inflammatory syndrome (IRIS) may present as new or worsening cavitation. We present an HIV-infected infant in whom TB infection and subsequent cavitation of the Ghon focus appeared to coincide with immune reconstitution due to highly active antiretroviral therapy (HAART). TB-IRIS in response to infection that occurs after starting HAART has not previously been described.
\end{abstract}

Primary pulmonary tuberculosis (TB) in children typically forms a Ghon complex visible on the chest radiograph as perihilar or paratracheal lymphadenopathy with or without an area of parenchymal infiltrate (Ghon focus). Cavitation or breakdown of lung parenchyma is significantly more common in HIV-infected than HIVuninfected children. The determinants of cavitation in children are not well defined. In adults, cavitary disease is common and is thought to be due to a delayed hypersensitivity response to the TB bacilli. New or worsening cavitation has been described as a presenting feature of TB-related immune reconstitution inflammatory syndrome (TB-IRIS) in adults after starting highly active antiretroviral therapy (HAART).

We present a case of cavitation of the Ghon focus in a 7month-old girl started on HAART at 2 months of age. We explore the pathogenesis of her cavitation, particularly focusing on two possibilities: an exaggerated immune response such as TB-IRIS, or an inadequate immune response due to incomplete immune reconstitution.

\section{CASE REPORT}

The patient's mother was identified as HIV-infected through participation in the Prevention of Mother to Child Transmission Program in the Western Cape, South Africa. Despite the mother having received antenatal zidovudine (ZDV) for 4 weeks, the infant's HIV DNA polymerase chain reaction (PCR) test was positive at 6 weeks of age. The viral load at that time was 43200 (log 4.64) copies per ml. The infant received HAART (ZDV, lamivudine and lopinavir/ritonavir) from 8 weeks of age through participation in the Children with HIV Early Antiretroviral therapy (CHER) trial. Compliance was confirmed with monthly pharmacy calculations. The baseline CD4 count was 908 cells/ $\mu$ l (CD4\% 22.9\%), rising to 1214 cells/ $\mu \mathrm{l}$ (31.4\%) after 3 months on HAART.

Five months after initiating HAART (i.e. at 7 months of age) the patient was hospitalised for an acute respiratory illness. Although she had no known TB contact, her weight had dropped significantly over the preceding month (Fig. 1). Her CD4 count had dropped acutely to 762 cells/ $\mu \mathrm{l}(23.6 \%)$. A chest radiograph showed hilar lymphadenopathy with left lower lobe opacification, and a single large cavity in the same region (Fig. 2). These changes were not present on baseline chest radiographs done at 2 months of age. Mycobacterium tuberculosis, fully susceptible to isoniazid and rifampicin, was cultured from a gastric aspirate. She was started on a standard three-drug TB regimen (isoniazid, rifampicin and pyrazinamide) with additional ritonavir added to her antiretroviral treatment, and thereafter displayed good catch-up growth (Fig. 1). A plasma HIV RNA level done at 14 months of age was below detectable limits, confirming adherence to HAART. The left lower lobe cavity had resolved completely after 9 months of TB treatment (i.e. at 16 months of age).

\section{DISCUSSION}

The subject's immune reconstitution had been reasonably successful. Three months after starting HAART (i.e. at 5 months of age) her CD4 count had risen from a

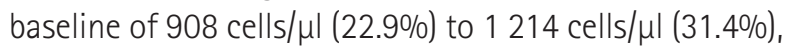




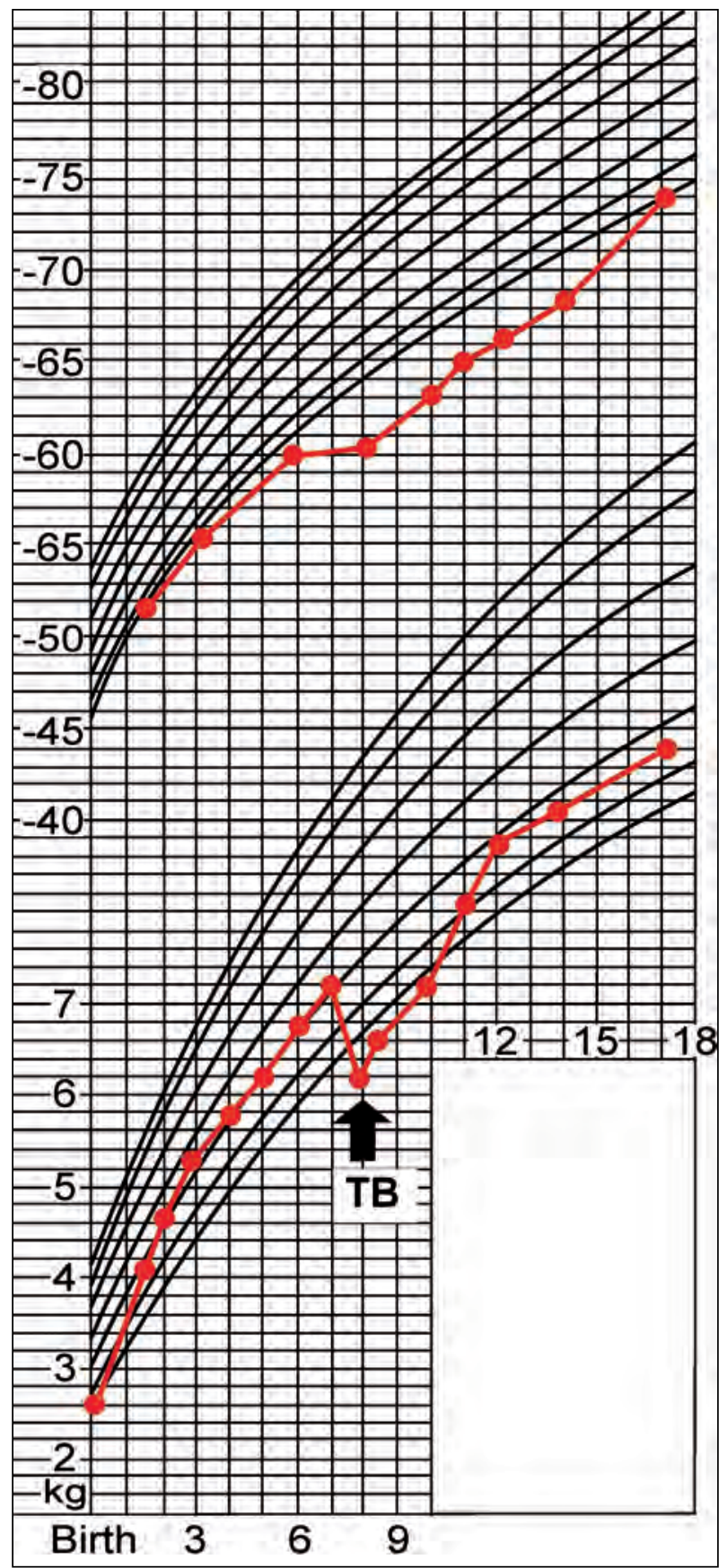

Fig. 1. Growth chart.

equivalent to the fifth centile for absolute CD4 counts among healthy black HIV-negative children between 3 and 6 months of age in Europe (1 200 cells/ $\mu$ l). ${ }^{1}$ However, it is possible that her immune response may have been marginally impaired since, although at the lower limit of normal, her absolute CD4 count was well below the median for age ( 2500 cells/ $/ \mu$ ).

The clinical evidence in this case suggests that TB infection occurred after the initiation of HAART. According to the growth curve, failure to thrive began 1 month before presentation (i.e. 4 months after the initiation of HAART). Wallgren documented that the development of the hypersensitivity response to TB in normal children (as shown by a positive tuberculin skin test or erythema nodosum) and the clinical manifestations of primary TB occur between 5 and 6 weeks after infection, although in exceptional cases, this may occur up to 8 weeks after

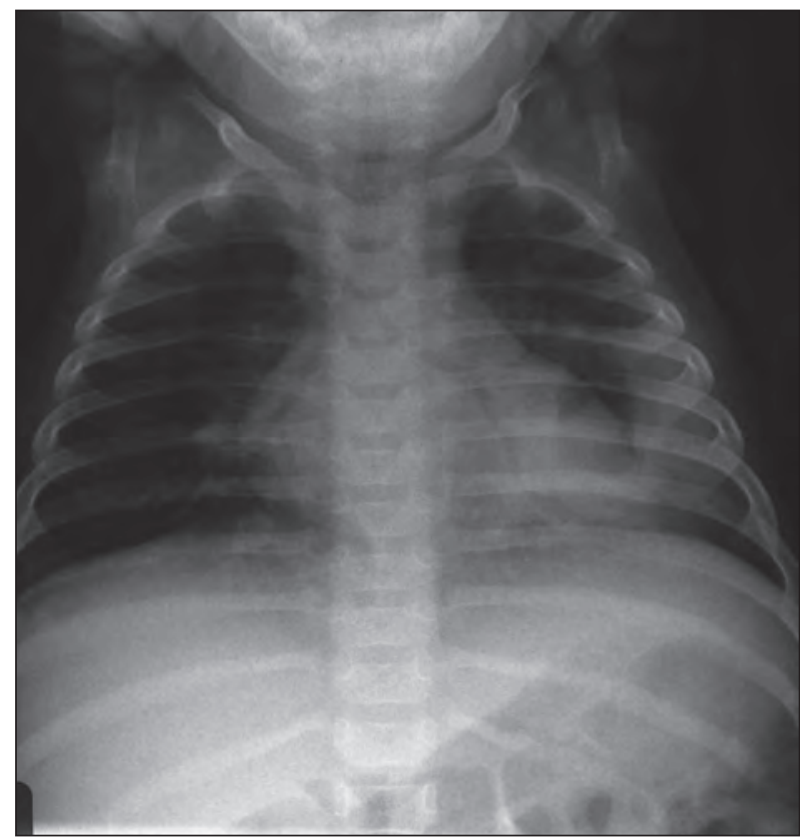

Fig. 2. Anteroposterior chest radiograph at 7 months of age.

infection. ${ }^{2}$ Therefore, according to Wallgren's 'timetable of tuberculosis' it is very unlikely that the manifestation of primary TB would have been delayed by 4 months.

In adults, new or worsening cavitation may be a presenting feature of TB-IRIS. Antimycobacterial activity is usually restored within 6 months of HAART in highly immunocompromised HIV-infected children. ${ }^{3}$ Consequently, clinical deterioration due to TB-IRIS generally occurs within 6 months of starting HAART. ${ }^{3}$ In this case,

\section{Acknowledgements}

Drs Helena Rabie and Heather Jaspan, Professors Peter Donald and Ben Marais of Stellenbosch University, and Dr Stephen Lawn of the University of Cape Town are thanked for constructive criticism and advice.

Financial support for CHER trial: National Institute of Allergy and Infectious Diseases (NIAID) of the US National Institutes for Health (NIH), through the Comprehensive International Program of Research on AIDS (CIPRA) network, grant number U19 Al53217. The Departments of Health of the Western Cape and Gauteng, South Africa and GlaxoSmithKline plc provided additional support. The content of this publication does not necessarily reflect the views or policies of NIAID, nor does mention of trade names, commercial projects, or organisations imply endorsement by the US Government.

The study was also conducted as an Investigational New Drug (IND) Number: IND 71,494 under the supervision of the Food and Drug Administration.

Contribution of authors: Dr Steve Innes prepared the manuscript. Professors Mark Cotton and Simon Schaaf personally supervised the development of the material. 
symptoms began 4 months after starting HAART. This case satisfied the paediatric IRIS working group consensus criteria for the diagnosis of TB-IRIS in children. ${ }^{4}$ Currently two forms of TB-IRIS have been described: acute unmasking of pre-existing TB infection, and paradoxical worsening of known pulmonary TB disease. ${ }^{4}$ Both 'unmasking' and 'paradoxical' IRIS occur in response to TB bacilli present in the lung parenchyma before the initiation of HAART.

The pathogenesis of IRIS is poorly understood but is related to the recovery of the Th-1 immune response following successful suppression of viral replication by HAART. ${ }^{5}$ The newly reconstituted Th- 1 system then reacts aggressively to antigens of dead and living organisms accumulated during immune suppression. This exaggerated (or possibly dysregulated) immune response causes local tissue damage, resulting in the typical manifestations of IRIS. There is some evidence that the recovery of M. tuberculosis-specific Th- 1 cells occurs more slowly than the recovery of other specific Th- 1 responses. ${ }^{6}$ This may result in a delay in the manifestation of TB-IRIS after the initiation of HAART.
In our case, TB infection most likely occurred after the initiation of HAART. Therefore our case cannot definitively be included in either the paradoxical or the unmasking category of TB-IRIS. We question whether this case may represent a third, previously unrecognised form of TBIRIS where exposure to a new pathogen may result in an exaggerated immunological response due to dysregulated immune recovery in the early phase of HAART. In young infants, it is possible that prior exposure to bacille Calmette-Guérin immunisation at birth may prime the immune system for an IRIS-like response on exposure to M. tuberculosis during the early phase of HAART. ${ }^{3}$

REFERENCES

European Collaborative Study. Age-related standards for total lymphocyte, CD4 and CD8 T-cell counts in children born in Europe. Pediatr Infect Dis J 2005; 24: 595600.

2. Wallgren A. The timetable of tuberculosis. Tubercle 1948; 29: 245-251.

3. Kampmann B, Tena-Coki GN, Nicol MP, Levin M, Eley B. Reconstitution of antimycobacterial immune responses in HIV-infected children receiving HAART AIDS 2006; 20(7): 1011-1018.

4. Boulware DR, Callens S, Pahwa S. Pediatric HIV immune reconstitution inflammatory syndrome. Curr Opin HIV AIDS 2008; 3: 461-467.

5. Ulrichs T, Moody DB, Grant E, Kaufmann SH, Porcelli SA. T-cell responses to CD1presented lipid antigens in humans with Mycobacterium tuberculosis infection Infect Immun 2003: 71(6): 3076-3087.

6. Schluger NW, Perez D, Liu YM. Reconstitution of immune responses to tuberculosis in patients with HIV infection who receive antiretroviral therapy. Chest 2002; 122(2): 597-602.

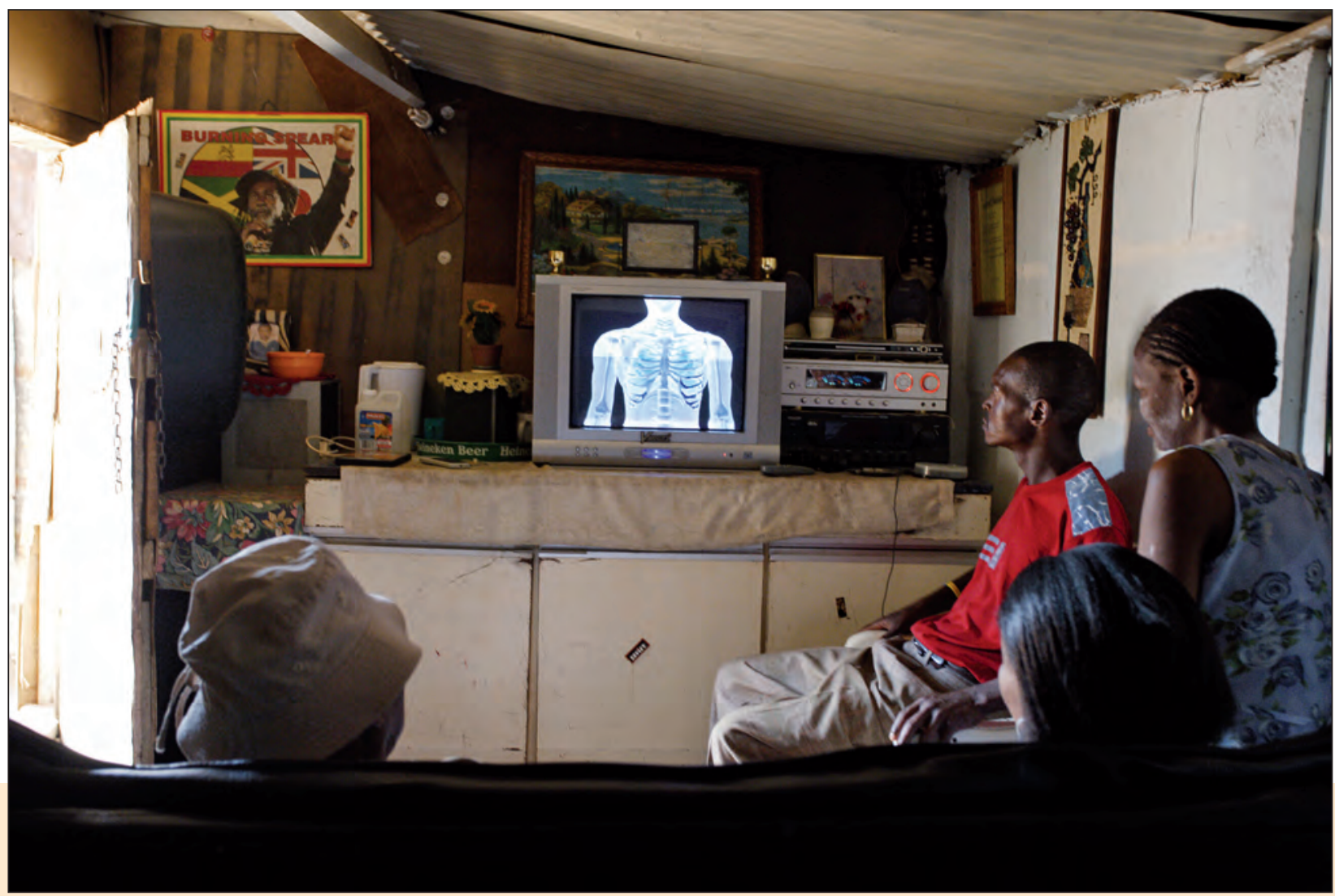

The South African Red Cross shows a Siyayinqoba educational video about TB and HIV to a family in Khatlehong. There are eight people living in the home. Their father died of TB last year after being infected for the third time. The daughter currently has MDR$T B$ and is on antiretrovirals. She was bedridden but is starting to feel better and able to walk around the house. Her brother is also showing symptoms of TB, and during this visit the Red Cross managed to convince him to be tested. 